COMMUNICATIONS IN

ANALYSIS AND GEOMETRY

Volume 14, Number 4, 725-764, 2006

\title{
Dehn filling and asymptotically hyperbolic Einstein manifolds
}

\author{
Gordon CRAig
}

\begin{abstract}
In this article, we extend Anderson's higher dimensional Dehn filling construction to a large class of infinite-volume hyperbolic manifolds. This gives an infinite family of topologically distinct asymptotically hyperbolic Einstein manifolds with the same conformal infinity. The construction involves finding a sequence of approximate solutions to the Einstein equations and then perturbing them to exact ones.
\end{abstract}

\section{Introduction}

In this article, we will describe a construction to generate infinitely many non-homotopic asymptotically hyperbolic Einstein (AHE) metrics with a shared conformal infinity. These are obtained by "capping off" or filling in the cusps of certain complete hyperbolic manifolds. The construction is based on Anderson's Dehn filling result for finite-volume hyperbolic manifolds [1], and we will refer readers to that paper where it is appropriate.

We will begin by providing background to this result and discussing its significance. After a brief sketch of the proof, the rest of this paper is devoted to the details.

Roughly speaking, asymptotically hyperbolic ( $\mathrm{AH})$ manifolds are complete Riemannian manifolds with an ideal boundary at infinity. The canonical example is the Poincaré ball with its sphere at infinity. For this to work, the underlying differential manifold must be a smooth, compact manifold with boundary.

Definition 1.1. Let $\bar{M}$ be a compact manifold with nonempty boundary. A smooth function $\rho$ is said to be a defining function for $\partial M$ iff

$$
\rho: \bar{M} \longrightarrow[0, \infty)
$$

satisfies $\rho(p)=0$ iff $p \in \partial M$ and $d \rho \neq 0$ on $\partial M$.

(c) 2006 Gordon Craig 
Then, we have

Definition 1.2. A complete metric $g$ on $M=\operatorname{int}(\bar{M})$ is said to be conformally compact iff there exists a defining function $\rho$ for $\partial M$ such that $\bar{g}=\rho^{2} g$ extends to a metric on $\bar{M}$.

As the name suggests, AH manifolds have curvature decaying to -1 at infinity. This is almost equivalent to conformal compactness, but we need to assume a small amount of regularity on the boundary. In what follows, quantities with a bar over them will be measured with respect to the compactified metric $\bar{g}$, and ones without bars will be measured with respect to the metric $g$ on $M=\operatorname{int}(\bar{M})$.

Definition 1.3. Consider a conformally compact metric $g$ on $M$. If there exists a defining function $\rho$ such that $|\bar{\nabla} \rho| \equiv 1$ on $\partial M$, then we say that $(M, g)$ is $\mathrm{AH}$.

The reason for this terminology is that in this case, the sectional curvatures of $(M, g)$ tend uniformly to $-1[2]$.

AH manifolds are a natural class of noncompact manifolds to work with because they have a nice structure at infinity; their curvature is tending toward a constant, and via the compactification $\bar{g}$, they have a "boundary metric" at infinity. Since this metric is determined by the choice of the function $\rho$, it only makes sense to speak of a boundary conformal class. This conformal class is known as the conformal infinity of the complete manifold $(M, g)$.

Given an $\mathrm{AH}$ manifold, it is natural to want to put a canonical $\mathrm{AH}$ metric on it. In two or three dimensions, the natural choice is a hyperbolic metric. In higher dimensions, however, hyperbolic metrics generalize in two ways: to hyperbolic metrics and to negatively curved Einstein metrics (constant negative Ricci curvature.) If $n>3$, the curvature tensor has more components than the metric, so prescribing sectional curvature becomes more difficult. On the other hand, the Ricci tensor has the same rank as the metric. So Einstein metrics are natural candidates to be canonical from an algebraic/PDE point of view. Accordingly, we define:

Definition 1.4. An AH manifold with constant Ricci curvature is called an (AHE) manifold.

AHE manifolds are sometimes referred to as Poincaré-Einstein manifolds. There has been a great deal of interest in AHE metrics recently due 
to their applications to physics [3-5]. Physicists are particularly interested in the correspondence between AHE metrics and their conformal infinities. From a more analytic/geometric perspective, this correspondence can be thought of as a geometric Dirichlet problem (although a priori the topology of the filling manifold could be undetermined).

Anderson has worked extensively on this correspondence in dimension 4 (c.f. the surveys $[4,6]$ and for more details $[2,7,29]$ ). In this case, there is generally not a bijective correspondence between AHE metrics and their conformal infinities, but under certain geometric conditions on the boundary, there are only finitely many AHE manifolds bounded by a given conformal class. This is not always the case, however; in [7], Anderson constructs infinitely many AHE metrics bounded by the same conformal class. He then shows in [7] that in dimension 4, any such collection has a limit point which is an AHE manifold with cusps (i.e., an Einstein metric whose ends are either conformally compact or of finite volume). Furthermore, this limit point has the same conformal infinity as all the elements in the set.

Under some fairly natural conditions on these limit manifolds, it is possible to show (still in four dimensions) that these limit manifolds are actually hyperbolic [7]. This suggests a natural question: given a hyperbolic manifold whose ends are either conformally compact or cusps, is there a sequence of AHE metrics with the same conformal infinity converging toward it? This is indeed the case in three dimensions (where AHE metrics are hyperbolic), although the methods used in this case come from hyperbolic geometry and cannot be applied to Einstein manifolds. Anderson has recently developed a cusp closing technique for finite-volume hyperbolic manifolds, generalizing Thurston's Dehn filling result to higher dimensions (c.f. [1]). His construction leads to infinite families of topologically distinct compact Einstein manifolds.

Our main result applies Anderson's cusp closing construction to generate a host of AHE metrics with the same conformal infinity:

Theorem 1.5. Let $\left(N^{n}, g\right),(n>2)$, be a complete geometrically finite hyperbolic manifold, all of whose cusps have toric cross sections. Then, it is possible to close the cusps to obtain infinitely many metrically distinct AHE manifolds, all of which have the same conformal infinity as the original one. If the hyperbolic manifold $N^{n}$ is nonelementary, then this procedure gives infinitely many homotopy types. If $n>3$, these AHE metrics are nonhyperbolic. 
It is possible to obtain a large class of manifolds satisfying the hypotheses of this theorem by taking Maskit combinations of complete hyperbolic cusps along hyperplanes. (c.f. [8] for this and also for some background on nonelementary and geometrically finite hyperbolic manifolds.) In dimension 3, our construction is simply a PDE proof of Thurston's Dehn-filling theorem, and so the manifolds we obtain are already well known [9]. In higher dimensions, however, it gives new examples of complete negatively curved Einstein manifolds.

From the point of view of the Dirichlet problem for AHE manifolds, we may restate our main result as follows:

Proposition 1.6. Let the conformal class $[\gamma]$ be the conformal infinity of a nonelementary geometrically finite hyperbolic manifold, whose ends are all either expanding or are toric cusps, and which has at least one cusp. Then, there are infinitely many topologically distinct AHE manifolds whose conformal infinity is $[\gamma]$.

It is interesting to note that all of the filling manifolds which we construct for $[\gamma]$ have the same Euler characteristic. If a conformal class $[\gamma]$ on a three-manifold includes a metric of positive scalar curvature, then the only way that it can bound infinitely many AHE four-manifolds is if their Euler characteristics are unbounded [2].

The proof of the main result follows Anderson's proof in [1]: we construct a sequence of approximate solutions to the Einstein equations (i.e., metrics whose Ricci curvature is tending toward some constant) and then perturb the metrics to an exact solution. This basic gluing procedure is quite common in geometric analysis, c.f., for example, [10-13].

The choice of approximate solutions is the main stumbling block in this procedure. Although in theory it is easy to prescribe Ricci curvature, since the Ricci tensor is of the same rank as the metric, in practice it is extremely difficult, because we must find explicit solutions to a coupled system of nonlinear PDEs. The construction of the approximate solutions requires the use of a very special (explicit) family of metrics, as we shall see below.

For the perturbation argument, we will be using a functional $\Phi$, and metrics which satisfy $\Phi(g)=0$ will be Einstein. Then, we will have a sequence of approximate solutions $g_{n}$ such that $\Phi\left(g_{n}\right) \longrightarrow 0$.

It turns out that sequences of approximate solutions degenerate, and so we cannot use a limiting argument to obtain our exact solution. On the other hand, the linearization of $\Phi$ at each metric $g_{n}$ is invertible. Thus, we could hope to use the inverse function theorem to invert $\Phi$ in a neighborhood 
of $\Phi\left(g_{n}\right)$. Since $\Phi\left(g_{n}\right) \longrightarrow 0$, we can hope that for $n$ large enough, one of these neighborhoods will contain 0 , which will give us a metric $g$ such that $\Phi^{-1}(0)=g$. Invertibility of $\Phi$ near $\Phi\left(g_{n}\right)$ is not enough to insure this, however. We could have a situation in which the region on which $\Phi$ is invertible shrinks as $n \longrightarrow \infty$, and so 0 never lies in this region.

Thus, we need to get a control on the size of the balls on which we can invert. If this is the case, then for $n$ large enough, we can perturb $g_{n}$ to a metric satisfying $\Phi(g)=0$. We obtain this uniform control by bounding the linearization of $\Phi$.

There is no need to assume any sort of nondegeneracy hypotheses on the original hyperbolic metric. (Roughly speaking, nondegeneracy means that a metric is a regular point for the Einstein operator. This is important if we want to apply an inverse function theorem, as we will.) We will actually see that our approximate solutions are nondegenerate as a consequence of our main estimate (4.1).

The main difference between our construction and Anderson's is that our approximate solutions are noncompact, due to the presence of the expanding ends. This introduces some technical difficulties in our analysis, but by construction, we have very strong control over the expanding ends, since our metrics have fixed conformal infinities.

As we mentioned above, in [7], Anderson showed that AHE manifolds with cusps which satisfy certain relatively natural conditions are in fact hyperbolic. This leads to another interesting question: are there any nonhyperbolic AHE manifolds with cusps? Our main result allows us to construct non-hyperbolic examples by closing the cusps on a hyperbolic manifold with several cusps and then reopening one of them.

Theorem 1.7. Given a nonelementary hyperbolic manifold $N^{n}, n>3$, with at least one expanding end and at least two cusps with toric crosssections, and no ends of another type, it is possible to construct infinitely many nonhomotopic nonhyperbolic Einstein manifolds which have at least one finite-volume end (i.e., a cusp) and whose infinite-volume ends are AH. Furthermore, their expanding ends have the same conformal infinities as $N^{n}$.

Let us now set some of our notation and conventions. From now on, all manifolds will be assumed to be complete and $\mathrm{AH}$, unless otherwise stated. Pointwise norms and inner products will be denoted by $|h|$ and $(f, h)$, respectively, while global ones will be denoted by $\|h\|$ and $\langle f, h\rangle . K$, ric, $z$ and $s$ will represent the sectional, Ricci, trace-free Ricci and scalar curvatures. $i(M)$ will denote the injectivity radius of $M . n$ will be reserved 
for the dimension the manifold $M$ and will always be strictly greater than 2. The curvature operator is defined as

$$
R(X, Y) Z=\nabla_{Y} \nabla_{X} Z-\nabla_{X} \nabla_{Y} Z-\nabla_{[X, Y]} Z
$$

for any three $X, Y, Z \in T M$. Our Laplacians will have negative spectrum, and so $\Delta_{S^{1}}=-d^{2} / d \theta^{2}$. This is the so-called "Geometer's Laplacian." We will often drop subscripts to improve readability if this will not lead to any confusion.

This article will be organized as follows: in Section 2, we construct our approximate solutions and discuss some of their properties. In Section 3, we will define our function spaces and operator and then in Section 4, we obtain a uniform control over the operator $D \Phi$ on all the approximate solutions. Finally, in Section 5, we wrap things up by perturbing our approximate solutions to exact ones and discuss some points of interest, including the proof of Theorem 1.7.

\section{Construction of approximate solutions}

In this section, we construct our approximate solutions and discuss some of their topological properties. We want to fill in the cusps of our hyperbolic manifold, and so we will be cutting them off and filling them in with solid tori. Topologically, this construction is easy. Even metrically it is not too hard, assuming that we do not require anything of the filled manifolds. But we want our filled manifolds (our approximate solutions) to have Ricci curvature close to a constant. This turns out to be much more difficult.

We will be filling each cusp separately. So we will only need to explain the procedure on one of them. By assumption, all of our cusps look like

$$
g_{C}=\rho^{-2} d \rho^{2}+\rho^{2} g_{T^{n-1}} ; \quad \rho_{0}>\rho>0
$$

Note that as $\rho \rightarrow 0$, the $T^{n-1}$ 's are collapsing. Without loss of generality, we can assume that $\rho_{0} \geq 1$ by rescaling the $\rho$ parameter. This will give us a metric of the same form, but with a rescaled $T^{n-1}$. Let us cut off the cusp at the torus $\rho=1$ and discard the region $0<\rho<1$. Then, we are faced with the task of attaching something to boundary torus $T_{0}$ in such a way that the metric on the glued manifold is smooth. Note that $T_{0}$ 's metric is the flat metric $g_{T^{n-1}}$.

For this construction to work, we will need to use a sequence of filling manifolds which are hyperbolic near their boundary and whose trace-free 
Ricci curvature tends toward zero. We will use members of a family of AHE metrics on $D^{2} \times T^{n-2}$. We can obtain our filling manifolds by truncating these at some fixed distance and then perturbing the metric near the boundary to make it hyperbolic. The perturbations will get smaller as we go further out, since the manifold is AH. We will start by discussing these filling manifolds.

Consider the following metric on $D^{2} \times T^{n-2}$;

$$
g_{\mathrm{BH}}=(V(r))^{-1} d r^{2}+V(r) d \theta^{2}+r^{2} g_{T^{n-2}},
$$

where $g_{T^{n-2}}$ is an arbitrary flat metric on the $(n-2)$-torus, and we use coordinates $(r, \theta)$ for the disk, with $r \geq r_{+}>0$ and $\theta \in[0, \beta]$. (Note that this means that the locus $r=r_{+}$is the center of the disk, and so there is a coordinate singularity there.)

We will specify the values of the parameters $r_{+}$and $\beta$ and the exact form of $V(r)$ below, but first let us calculate the curvatures of these metrics in terms of the function $V(r)$. We will start by setting up an orthonormal basis for these metrics: let $e_{1}=\sqrt{V} \partial_{r}, e_{2}=1 / \sqrt{V} \partial_{\theta}$ and $e_{j}=1 / r \partial_{\phi_{j}}$, where the $\partial_{\phi_{j}}, 3 \leq j \leq n$ are orthonormal basis for the $T^{n-2}$. A straightforward calculation shows that the $e_{i}$ diagonalize the curvature tensor and that the corresponding sectional curvatures are

$$
\begin{aligned}
& K_{12}=-\frac{V^{\prime \prime}}{2} \\
& K_{1 j}=K_{2 j}=-\frac{V^{\prime}}{2 r} \quad j>2 \\
& K_{i j}=-\frac{V}{r^{2}} \quad i, j>2 .
\end{aligned}
$$

Now, let

$$
V(r)=r^{2}-2 m r^{3-n}
$$

Using the same basis as above, we have

$$
\begin{aligned}
& K_{12}=-1+\frac{(n-3)(n-2) m}{r^{n-1}} \\
& K_{1 j}=K_{2 j}=-1-\frac{(n-3) m}{r^{n-1}} \\
& K_{i j}=-1+\frac{2 m}{r^{n-1}},
\end{aligned}
$$

where once again $i, j$ are assumed to be greater than 2 . 
Another straightforward calculation shows that this metric is Einstein with scalar curvature $-n(n-1)$ and AH. (If $n=3$, it will be hyperbolic.) We have yet to specify the range of the $r$ parameter, but it is clear that the metric is well defined for large enough $r$, so it makes sense to speak of its local and asymptotic properties [30].

If $m=0$, we get a hyperbolic cusp metric

$$
g_{C}=r^{-2} d r^{2}+r^{2} g_{S^{1} \times T^{n-2}} .
$$

This metric will be complete if we let $r \in(0, \infty)$.

On the other hand, if $m>0$ and $n>3$, we get a nontrivial Einstein metric. These metrics are called $T^{n-2}$ Anti-deSitter Black Hole metrics. They will be complete provided we let $r \geq r_{+}=(2 m)^{1 /(n-1)}$ and $\theta \in\left[0, \beta_{m}\right]$, where

$$
\beta_{m}=\frac{4 \pi}{(n-1) r_{+}}
$$

[7]. Note that the locus $\left\{r=r_{+}\right\}$is a flat totally geodesic $T^{n-2}$. By analogy with the core geodesics in hyperbolic Dehn surgery [14], we call this a core torus.

Now recall that we introduced these manifolds because we want to glue them into a cusp. They have the correct topological and local geometric properties to work. The first choice we could make would be to cut off one of the black hole metrics above at some large $r$ and then perturb it to make it hyperbolic near the boundary. The problem is that we cannot fix the global geometry near the boundary; although we can choose the metric on the $T^{n-2}$, the boundary metric will necessarily be the product of this flat metric and a large $S^{1}$, since the size of the $S^{1}$ factor is determined by $r$.

To resolve this difficulty, we will exploit the large isometry group of these metrics to take a quotient with the desired boundary. Below, we shall use the term "black hole metric" to refer to any metric on $D^{2} \times T^{n-2}$ which has the same universal cover as $g_{\mathrm{BH}}$.

Proposition 2.1. Suppose we have an $S^{1} \times \mathbb{R}^{n-2}$-invariant metric on $D^{2} \times \mathbb{R}^{n-2}$. Let $T_{0}$ be some flat $(n-1)$-torus, and let $\sigma \subset T_{0}$ be a simple closed geodesic such that

$$
L(\sigma)=L\left(\partial D^{2}\right)
$$


Then, $\exists \Gamma_{0} \subset \operatorname{Isom}\left(D^{2} \times \mathbb{R}^{n-2}\right)$ such that

$$
\frac{\left(D^{2} \times \mathbb{R}^{n-2}\right)}{\Gamma_{0}} \simeq M_{\text {fill }}
$$

is a solid torus with $\partial M_{\text {fill }}=T_{0}$.

Proof. We have

$$
T_{0}=\frac{\mathbb{R}^{n-1}}{\Gamma}
$$

where $\Gamma$ is some $(n-1)$-dimensional group of translations of $\mathbb{R}^{n-1}$. Since $\sigma$ is closed and simple, the translation induced by $\sigma$ is a generator for $\Gamma$. We can find elements $\gamma_{i} \in \mathbb{R}^{n-1}$ such that the set $\left\{\sigma, \gamma_{1}, \ldots, \gamma_{n-2}\right\}$ forms a set of generators for $\Gamma$. Let us denote the subgroup of $\pi_{1}\left(T_{0}\right)$ generated by $\sigma$ by $\langle\sigma\rangle$. Then, since $\pi_{1}\left(T_{0}\right)$ is Abelian, $\langle\sigma\rangle$ is normal, which implies that the covering map

$$
p: \mathbb{R}^{n-1} \longrightarrow T_{0}
$$

splits as $p=p_{1} \circ p_{2}$, where

$$
p_{2}: \mathbb{R}^{n-1} \longrightarrow \frac{\mathbb{R}^{n-1}}{\langle\sigma\rangle} \cong S^{1} \times \mathbb{R}^{n-2}
$$

and

$$
p_{1}: S^{1} \times \mathbb{R}^{n-2} \longrightarrow \frac{\left(S^{1} \times \mathbb{R}^{n-2}\right)}{\Gamma_{0} \cong T_{0}},
$$

where $\Gamma_{0}=\Gamma /\langle\sigma\rangle$.

Now, say we have an $S^{1} \times \mathbb{R}^{n-2}$-invariant metric on $D^{2} \times \mathbb{R}^{n-2}$ and that the length of $\partial D^{2}$ is $L(\sigma)$.

We will use the above remark to construct a quotient of this metric with boundary $T_{0}$. We can describe this quotient in terms of coordinates $\left(r, \theta, \phi_{3}, \ldots, \phi_{n-2}\right)$, where the $\phi_{i}$ 's are the standard coordinates on $\mathbb{R}^{n-2}$. Define an isometric action of $\Gamma_{0}$ on $D^{2} \times \mathbb{R}^{n-2}$ by keeping $r$ fixed and acting on the $S^{1} \times \mathbb{R}^{n-2}$ coordinates. The boundary of this quotient will certainly be $T_{0}$, and it is clear that there are no fixed points on $r=0$ since no element maps $\sigma$ to itself except the identity, and so the quotient is indeed a manifold. 
Note that the reason that this works is that we were able to split off the $\langle\sigma\rangle$ from the rest of $\Gamma$ and then fill it in with a disk. The basic point is that when we project the other generators onto the core $\mathbb{R}^{n-2}$, they cannot be zero, or else they would be parallel to $\sigma$. If one thinks about the threedimensional case, one can picture the universal cover as being a tubular neighborhood of a geodesic in hyperbolic space. Then, $\sigma$ would be the boundary of a disk perpendicular to the core geodesic. One can obtain the torus $T^{2}$ by taking the quotient of the cylinder by $\langle\gamma\rangle$, where $\gamma$ is some composition of a translation and a rotation. The only way that this action will not extend to the core geodesic is if $\gamma$ has no translation component. But this is impossible if the quotient of the boundary is to be a torus.

Now we will get an appropriate metric on this quotient. All we need is an $S^{1} \times \mathbb{R}^{n-2}$-invariant metric on $D^{2} \times \mathbb{R}^{n-2}$. Since we want an Einstein metric, we will take the universal cover of the $T^{n-2}$-black hole metrics, slightly altered near the boundary. It turns out that the value of $m$ is irrelevant to the local geometry of these [7], and so we will set $m=1$.

Let

$$
R=\frac{L(\sigma)}{\beta_{1}}
$$

and define

$$
\widetilde{g_{\text {fill }}}=V(r)^{-1} d r^{2}+V(r) d \theta^{2}+r^{2} g_{\text {Eucl }} ; r \in\left[r_{+}, R\right), \theta \in\left[0, \beta_{1}\right),
$$

where $V(r)=r^{2}-2 \chi(r) / r^{n-3}, \quad \chi(r)=1$ for $r<R-2, \quad \chi(r)=0$ for $r>R-1$.

Now note that for $r>R-1$,

$$
\widetilde{g_{\text {fill }}}=r^{-2} d r^{2}+r^{2}\left(d \theta^{2}+g_{\text {Eucl }}\right)
$$

By taking the quotient, we will get the metric

$$
g_{\text {fill }}=r^{-2} d r^{2}+r^{2} \frac{g_{T_{0}}}{R^{2}}
$$

on $r>R-1$.

By the change of coordinates

$$
\rho=\frac{r}{R}
$$

we get a metric which is identically equal near its boundary to the hyperbolic cusp metric which we are trying to fill in. In the event that we have $k$ toric 
cusps, we can cut off each one and perform this procedure on a geodesic $\sigma^{i}$ in each boundary torus. We then obtain a manifold $\left(M_{\sigma}, g_{\sigma}\right)$, where $\sigma$ is the ordered $k$-tuple of geodesics $\left(\sigma^{i}\right)_{1 \leq i \leq k}$.

We say that $M_{\sigma}$ is a Dehn filling of $N$, again by analogy with the threedimensional case. The size of the Dehn filling is defined to be

$$
|\sigma|=\min _{i} L\left(\sigma^{i}\right)=\min _{i}\left(R_{\sigma^{i}} \beta_{1}\right) .
$$

This is well defined, since we fix the boundary tori at the beginning.

In three dimensions, we no longer have these black hole metrics. In fact, the only candidate for the glued-in metric is a quotient of hyperbolic space. But there are many hyperbolic three-manifolds bounded by 2-tori; we can repeat the above Dehn filling on the tubular neighborhood of a geodesic in hyperbolic 3 -space. The analysis below then goes through analogously to the higher dimensional case.

We end this section with a few remarks on the topology of the $M_{\sigma}$ 's. We start with the Gromov-Thurston $2 \pi$-theorem. (For an elementary proof, c.f. $[15])$.

Proposition $2.2[16]$. If $|\sigma|>2 \pi$, then $M_{\sigma}$ admits a nonpositively curved metric such that the core tori are completely geodesic and whose sectional curvature is strictly negative off the core tori.

Thus, we have:

Proposition 2.3. For $|\sigma|>2 \pi, M_{\sigma}$ is a $K(\pi, 1)$, and every noncyclic Abelian subgroup of $\pi_{1}\left(M_{\sigma}\right)$ is carried by one of the core tori.

Proof. (Adapted from Theorem 6.3.9 of [17]). The first statement follows directly from the fact that $M_{\sigma}$ admits a metric of nonpositive sectional curvature. To prove the second one, consider the action of $\pi_{1}\left(M_{\sigma}\right)$ by isometries on $\widetilde{M_{\sigma}}$, the universal cover of $M_{\sigma}$, where $\widetilde{M_{\sigma}}$ is equipped with the lifted metric. Let $\alpha \in \pi_{1}\left(M_{\sigma}\right)$, and let $p \in \widetilde{M_{\sigma}}$ satisfy

$$
d(p, \alpha(p))=\inf _{x \in \widetilde{M}_{\sigma}} d(x, \alpha(x)) .
$$

Such a $p$ exists because $M_{\sigma}$ 's injectivity radius is nonzero and is large on the expanding ends of $M_{\sigma}$. Thus, any minimizing sequence for the function $d(x, \alpha(x))$ must project down to some compact subset of $M_{\sigma}$.

Since $\widetilde{M_{\sigma}}$ is simply connected and of nonpositive curvature, there is a unique geodesic $\gamma$ joining $p=\gamma(0)$ and $\alpha(p)=\gamma\left(t_{0}\right)$. Furthermore, $\alpha$ must 
fix $\gamma$. To show this, all we need to prove is that $\alpha^{2}(p)$ lies on $\gamma$. If it does not, then let $x=\gamma\left(t_{0} / 2\right)$ be the midpoint of the segment $\overline{p \alpha(p)}$ of $\gamma$. By assumption, the three points $x, \alpha(x)$ and $\alpha(p)$ cannot all lie on the same geodesic, and so we must have

$$
\begin{aligned}
d(x, \alpha(x)) & <d(x, \alpha(p))+d(\alpha(p), \alpha(x)) \\
& =\frac{t_{0}}{2}+d(p, x) \\
& =t_{0} \\
& =d(p, \alpha(p)),
\end{aligned}
$$

which is impossible, since $p$ minimizes $d(x, \alpha(x))$.

If $\alpha, \beta \in \pi_{1}\left(M_{\sigma}\right)$ commute, then $\alpha \circ \beta(p)=\beta \circ \alpha(p)$. So $\beta$ sends the geodesic segment $\overline{p \alpha(p)}$ to the geodesic segment $\overline{\beta(p) \beta(\alpha(p))}$. Furthermore, we can see that $\alpha$ must map the unique geodesic joining $p$ to $\beta(p)$ to the unique one joining $\alpha(p)$ and $\beta(\alpha(p))$. This gives a geodesic quadrilateral whose angles add up to $2 \pi$. By the Topogonov theorem, this quadrilateral must lie in a flat submanifold, and so we are done.

We will also need the following result:

Proposition 2.4. Let $N$ admit a nonelementary geometrically finite hyperbolic metric. Then $N$ admits only finitely many homotopy equivalence classes.

Proof. Let

$$
F: N \longrightarrow N
$$

be a homotopy equivalence. Given a hyperbolic metric $g$ on $N$, we can deform $F$ so that it fixes the conformal infinity of $(N, g)$. Then by Sullivan rigidity [8], $F$ can be represented by an isometry. But by [18], generic nonelementary geometrically finite hyperbolic metrics have only finitely many isometries.

We may now prove that our Dehn fillings give rise to infinitely many homotopy classes.

Proposition 2.5. Let $N$ admit a geometrically finite nonelementary hyperbolic metric, all of whose cusps are tori. Let $M_{\sigma}$ be obtained from $N$ by a Dehn filling. Then, there are only finitely many other Dehn fillings which have the same homotopy type as $M_{\sigma}$. 
Proof. We will simply adapt Anderson's proof from [1] to the infinite-volume case. The idea of the proof is to show that a homotopy equivalence

$$
F: M_{\sigma} \longrightarrow M_{\sigma^{\prime}}
$$

leads either to a nontrivial homotopy equivalence of the hyperbolic manifold $N$ or to a nontrivial isomorphism of the Dehn filling data. Since there are only finitely many members in either of these classes, we can conclude that there are only finitely many $M_{\sigma}$ 's in any homotopy class.

By Seifert-Van Kampen, we have that

$$
\pi_{1}\left(M_{\sigma}\right)=\frac{\pi_{1}(N)}{\left\langle\cup \sigma_{i}\right\rangle} .
$$

By Proposition 2.3, we know that for $|\sigma|$ sufficiently large, only the conjugacy classes of noncyclic Abelian subgroups of $\pi_{1}\left(M_{\sigma}\right)$ are carried by the core tori. Now, say that we have a homotopy equivalence

$$
F: M_{\sigma} \longrightarrow M_{\sigma^{\prime}}
$$

Then, $F_{*}$ must permute the cyclic subgroups carried by the essential tori. This in turn implies that $F$ must map neighborhoods of these tori onto each other. We can then use $F$ to define a map $G$ from the original hyperbolic manifold $N$ to itself such that $G$ fixes the conformal infinity of $N$, interchanges the cusps of $N$ and such that $G_{*}$ is an isomorphism of $\pi_{1}(N)$. Then, again by Sullivan rigidity, $G$ is a homotopy equivalence, of which there are only finitely many by the previous proposition. Now the homotopy equivalence $F$ must also preserve the Dehn filling data, and so necessarily we must have

$$
F_{*}\left\langle\sigma_{i}\right\rangle=\left\langle\sigma_{j}^{\prime}\right\rangle
$$

But given a cyclic group, there are only two elements which can generate it. Thus, there are only finitely many Dehn-filled manifolds homotopy equivalent to $M_{\sigma}$.

The proposition does not hold if we drop the hypothesis that $(N, g)$ is nonelementary; one can construct infinitely many nonisometric black hole metrics on the solid torus $D^{2} \times T^{n-2}$ with the same conformal infinity $T^{n-1}[7]$. These can also be thought of as Dehn fillings of the complete hyperbolic cusps

$$
g_{C}=r^{-2} d r^{2}+r^{2} g_{T^{n-1}} .
$$




\section{Analytic preliminaries}

Let us start by establishing the function spaces we will be using. To obtain good analytic properties, such as elliptic estimates and precompactness of bounded sequences, we will work with Hölder spaces. The precise definition of these spaces requires some care, as we will want to be able to compare spaces defined on a large class of manifolds.

Proposition 3.1. [19] Let $Q>1, C, i_{0}>0, k \in \mathbb{N}, 0<\alpha<1$. Then, there exists $\rho_{0}>0$ such that if $\| \nabla^{k-1}$ ric $\|_{L^{\infty}} \leq C$ and $i(M) \geq i_{0}$, then for any $x \in M$, the ball $B\left(x, \rho_{0}\right)$ has harmonic coordinates in which we have

$$
Q^{-1} I \leq g \leq Q I
$$

$$
\begin{aligned}
& \sum_{1 \leq|\beta| \leq k} \rho_{0}^{|\beta|} \sup _{y \in B\left(x, \rho_{0}\right)}\left|\partial^{\beta} g_{i j}(y)\right| \\
& \quad+\sum_{|\beta|=k} \rho_{0}^{k+\alpha} \sup _{y_{1}, y_{2} \in B\left(x, \rho_{0}\right)} \frac{\left|\partial^{\beta} g_{i j}\left(y_{1}\right)-\partial^{\beta} g_{i j}\left(y_{2}\right)\right|}{\left|y_{1}-y_{2}\right|^{\alpha}} \leq Q-1 .
\end{aligned}
$$

Here, we will fix $Q$ sufficiently close to 1 for the rest of this paper. We shall call the above coordinates $C^{k, \alpha}$-harmonic coordinates. Now, given $C>0, i_{0}>0$, we can define $(k, \alpha)$-Hölder norms on the class of manifolds with $\| \nabla^{k-1}$ ric $\|_{L^{\infty}}<C$ and $i(M)>i_{0}$. Start by choosing a locally finite collection of balls $B\left(x_{i}, \rho_{0}\right)$ with $(k, \alpha)$-harmonic coordinates which cover $M$ such that the balls $B\left(x_{i}, \rho_{0} / 4\right)$ are disjoint. (This is possible because the Ricci curvature is bounded.) Then define the $(k, \alpha)$-Hölder norm of $h \in S^{2}(M)$

$$
\begin{aligned}
\|h\|_{k, \alpha}=\sup _{x_{i}} & \left\{\sum_{1 \leq|\beta| \leq k} \rho_{0}^{|\beta|} \sup _{y \in B\left(x_{i}, \rho_{0}\right)}\left|\partial^{\beta} h_{i j}(y)\right|\right. \\
& \left.+\sum_{|\beta|=k} \rho_{0}^{k+|\alpha|} \sup _{y_{1}, y_{2} \in B\left(x_{i}, \rho_{0}\right)} \frac{\left|\partial^{\beta} h_{i j}\left(y_{1}\right)-\partial^{\beta} h_{i j}\left(y_{2}\right)\right|}{\left|y_{1}-y_{2}\right|^{\alpha}}\right\},
\end{aligned}
$$

where the supremum is taken over all the balls $B\left(x_{i}, \rho_{0}\right)$. We can then define the $C^{k, \alpha}$ topology on the space of metrics near $g_{0}$ by setting the norm of a 
metric $g$ near $g_{0}$ to be

$$
\left\|g-g_{0}\right\|_{k, \alpha}
$$

From here on out $i_{0}, C, k>2$ and $\alpha$ will all be fixed, and our reference metrics will be our approximate solutions $\left(M_{\sigma}, g_{\sigma}\right)$.

Now, it is clear that on open bounded sets $\Omega \subset M$, we will have that the inclusion of $C^{k, \alpha}(\Omega)$ into $C^{k, \alpha^{\prime}}(\Omega)$ is compact for $\alpha^{\prime}<\alpha$. Furthermore, by our control of the metric in these coordinates, we will have interior Schauder estimates for elliptic operators on bounded sets $\Omega \subset M[20]$.

Our analysis will take place on manifolds approximating the hyperbolic manifold whose cusps we will be closing. Thus, we need to take into account two types of noncompact behavior: moving down the cusps makes the injectivity radius arbitrarily small and the diameter of the end tend toward infinity, and moving out into the expanding end makes the volume tend toward infinity. We will deal with these issues separately. In all of these cases, we will need infinitely many coordinate charts. Thus, to be able to get uniform control over the entire manifold, we shall need to have coordinate charts which are "uniformly similar" in some sense; i.e., they must be defined on balls of approximately the same size and local geometry. We will also need weight functions to deal with the infinite volume of the expanding ends and the unbounded diameter of the filled ends.

The problem that we will encounter from the injectivity radius tending toward zero is that our coordinate charts will have to be made arbitrarily small as we move down the cusp. On the other hand, since the geometry is hyperbolic (or, as we shall see, very close to it), we can lift to a large enough cover and then calculate the norm on this cover.

Thus, we take Anderson's definition from [1]:

Definition 3.2. We say that a manifold has uniformly bounded local covering geometry if, given some fixed constant $i_{0}>0$, any ball $B\left(x, i_{0}\right)$ has a finite cover $\bar{B}\left(\bar{x}, i_{0}\right)$ with diameter less than 1 and $i(\bar{x}) \geq i_{0}$.

Then, define the modified $C^{k, \alpha}(M)$ norm $\tilde{C}^{k, \alpha}(M)$ to be the $C^{k, \alpha}(M)$ norm, with the norm being evaluated in $(k, \alpha)$-harmonic coordinates on a large enough cover if the injectivity radius is less than $i_{0}$.

Now, let us define the main space which we will be working with. Let $S^{2}(M)$ be space of smooth symmetric bilinear forms on $T M$.

Definition 3.3. Define $S^{k, \alpha}$ to be the completion of $S^{2}(M)$ with respect to the $\tilde{C}^{k, \alpha}(M)$ norm. 
Even though the space $S^{k, \alpha}$ is well defined for our noncompact manifolds, it is too large for our purposes, since it includes many forms over whose asymptotic behavior we have very little control. Furthermore, we do not want to change the conformal infinity of our approximate solution when we perturb it to an exact solution, and so we want our perturbation to vanish at infinity. Both of these considerations lead us to the following definition:

Definition 3.4. Let $\rho$ be a geodesic defining function, and let $r(x)=$ $\log (2 / \rho)$. For $\delta>0$, we define the $\delta$-weighted Hölder space $S_{\delta}^{k, \alpha}(M)$ to be

$$
\left\{u=\mathrm{e}^{-\delta r} u_{0} \mid u_{0} \in S^{k, \alpha}(M)\right\}
$$

If $u \in S_{\delta}^{k, \alpha}(M)$, define $\|u\|_{k, \alpha, \delta}=\left\|u_{0}\right\|_{k, \alpha}=\left\|\mathrm{e}^{\delta r} u\right\|_{k, \alpha}$.

Note that although this norm depends on our choice of $\rho$, the space $S_{\delta}^{k, \alpha}$ does not. $\rho$ is a geodesic defining function iff $|\bar{\nabla} \rho| \equiv 1$ in a neighborhood of $\partial M$. Such functions have the property that if $r=\log (2 / \rho)$, then

$$
|\nabla r|=|\bar{\nabla} \rho| \equiv 1
$$

in some neighborhood of $\partial M$. Thus, $r$ is a distance function outside some compact set. It always possible to construct such defining functions for $\mathrm{AH}$ metrics [21].

We then have the following analytic results.

Theorem $3.5[22]$. Let $\delta^{\prime}<\delta$ and $\alpha^{\prime}<\alpha$. Then the inclusion $S_{\delta}^{k, \alpha}(M) \rightarrow$ $S_{\delta^{\prime}}^{k, \alpha^{\prime}}(M)$ is compact.

We will be using Bochner-technique arguments. So we shall need to use forms which are square-integrable. The following lemma describes under which conditions this occurs.

Lemma 3.6 [22]. Let $(M, g)$ be AH. If $\delta>n-1 / 2$, then $S_{\delta}^{k, \alpha}(M) \subset$ $L^{2}\left(S^{2}(M)\right)$.

We have yet to deal with the fact that the diameter of the filling of each end is tending to infinity. We will once again modify our function spaces, but this time the reason for the modification will not be as clear as the two previous ones were. The reason for this is that the change we are making is specifically adapted to our particular problem. Because of this, we will 
wait until we actually use it to explain the motivation. The basic idea is that the diameter of each of the various filled-in ends is determined locally on the end and has nothing to do with those of the other ends. Thus, to obtain uniform bounds, we must weigh our function spaces with a different factor for each end.

To begin, consider any of our filled manifolds $M_{\sigma}$. For now, say we are in the filling region of the first cusp, so the $r$ coordinate lies in the interval $\left[r_{+}, R^{1}\right]$, where $R^{1}=L\left(\sigma^{1}\right) / \beta$. Let $r_{c} \in\left[r_{+}, R^{1}\right]$. Define the function

$$
\psi_{c}= \begin{cases}\frac{r_{c}}{R^{1}} & \text { if } r_{+} \leq r \leq r_{c} \\ \frac{r}{R^{1}} & \text { if } r_{c} \leq r \leq R^{1}\end{cases}
$$

Assuming $N$ has $l$ cusps, we may define such a function on each filled cusp. Let $r_{c}=\left(r_{c}^{j}\right)_{l=1}^{l}$ be a $l$-tuple such that $r_{c}^{j} \in\left[r_{+}, R^{j}\right]$. Then define $\phi_{c}$ to be equal to the corresponding $\psi_{c}$ on each filling region, and equal to 1 on the rest of the manifold, up to a smoothing at each gluing torus in such a way that its $\tilde{C}^{k, \alpha}$ norm is uniformly bounded. Note that no matter what our choices are, $\phi_{c}$ is bounded above by 1 , but it has no uniform positive lower bound. Note also that $\phi_{c}$ is constant near each core tori.

Definition 3.7. Let $k, \alpha$ and $\delta$ be as above. Consider the space $S_{\delta}^{k, \alpha}\left(M_{\sigma}\right)$. Let $r_{c}=\left(r_{c}^{j}\right)_{j=1}^{l} \quad$ satisfy $r_{c}^{j} \in\left[r_{+}, R^{j}\right]$, where $R^{j}=L\left(\sigma^{j}\right) / \beta$ is the $r$-coordinate of the $j$ th gluing torus in the coordinate system of the filling manifold. We define the modified $S_{\delta, r_{c}}^{k, \alpha}$ norm of $h \in S_{\delta}^{k, \alpha}\left(M_{\sigma}\right)$ to be

$$
\|h\|_{k, \alpha, \delta, r_{c}}=\left\|\phi_{c}^{-1} h\right\|_{k, \alpha, \delta} .
$$

Note that the spaces $S_{\delta, r_{c}}^{k, \alpha}$ and $S_{\delta}^{k, \alpha}$ are identical for every fixed $M_{\sigma}$, and that a uniform bound on the $S_{\delta, r_{c}}^{k, \alpha}$ norm of a sequence implies one on the $S_{\delta}^{k, \alpha}$ norm, regardless of what $r_{c}$ is. $r_{c}$ will be fixed below, in Section 4 .

In analogy to the $C^{k, \alpha}$ norm, we can define the $C_{\delta, r_{c}}^{k, \alpha}$ norm on metrics near our approximate solution $g_{\sigma}$ by setting the norm of $g$ to be

$$
\left\|g-g_{\sigma}\right\|_{k, \alpha, \delta, r_{c}}
$$

Note that any metrics within a finite distance of each other with respect to this norm must have the same conformal infinity.

We will now discuss the operator which we shall use. To eliminate trivial kernel arising from rescalings and diffeomorphisms, we will use the following 
operator instead of the trace-free Ricci curvature $z$.

$$
\begin{array}{r}
\Phi_{g_{\sigma}}: S_{\delta, r_{c}}^{k, \alpha}\left(M_{\sigma}\right) \longrightarrow S_{\delta, r_{c}}^{k, \alpha}\left(M_{\sigma}\right) \\
g \longmapsto \operatorname{ric}_{g}+(n-1) g+\delta_{g}^{*} B_{g_{\sigma}}(g),
\end{array}
$$

where $g_{\sigma}$ is the approximate solution and $B_{g_{\sigma}}$ is its associated Bianchi operator

$$
\begin{gathered}
B_{g_{\sigma}}: S^{2}(M) \longrightarrow \Omega^{1}\left(M_{\sigma}\right) \\
B_{g_{\sigma}}(h)=\delta_{g_{\sigma}} h+\frac{1}{2} d t r_{g_{\sigma}} h .
\end{gathered}
$$

It is straightforward to check that this is indeed a well-defined map between these spaces. Now the linearization of $\Phi$ at $g_{\sigma}$ is [23]

$$
D_{g_{\sigma}} \Phi(h)=\frac{1}{2} \Delta_{L} h+(n-1) h
$$

where the Lichnerowicz Laplacian $\Delta_{L}$ is defined as

$$
\begin{gathered}
\Delta_{L}: S^{2}(M) \longrightarrow S^{2}(M) \\
\Delta_{L} h=D^{*} D h+\text { ric } \circ h+h \circ \text { ric }-2 R(h),
\end{gathered}
$$

and $R$ is the action of the curvature tensor on $S^{2}(M)$ :

$$
\begin{aligned}
R h(X, Y) & =\operatorname{tr}(((W, Z) \mapsto h(R(X, W) Y, Z)) \\
& =\sum_{i=1}^{n} h\left(R\left(X, e_{i}\right) Y, e_{i}\right),
\end{aligned}
$$

where $\left\{e_{i}\right\}$ is an orthonormal basis with respect to the metric at which we are linearizing. Composition of symmetric bilinear forms is defined by using the metric to identify them with elements of $\operatorname{Hom}(T M, T M)$.

$D_{g_{\sigma}} \Phi$ is clearly elliptic, and so $\Phi$ is elliptic near $g_{\sigma}$. The relation between $\Phi_{g_{\sigma}}$ and Einstein metrics is described by the following lemma:

Lemma 3.8 [23]. Let $(M, g)$ be AH, with ric $_{g}<0$. If $\Phi_{g_{\sigma}}(g)=0$ and $\lim _{r \rightarrow \infty}\left|B_{g_{\sigma}}(g)\right|=0$, then $\operatorname{ric}_{g}=-(n-1) g$. In other words, $g$ is Einstein with scalar curvature $-n(n-1)$.

The reader may be concerned that we are only defining our operator $\Phi$ and our function spaces near some base metric $g_{\sigma}$. This is not an issue, since 
we are using a perturbation argument and thus will only be working in a neighborhood of the metric we wish to perturb.

We are now ready to show why the $M_{\sigma}$ of the previous section can be called approximate solutions.

We can see explicitly that

$$
\begin{aligned}
\left\|\Phi_{g_{\sigma}}\left(g_{\sigma}\right)\right\|_{k-2, \alpha, \delta, r_{c}} & =\left\|\operatorname{ric}_{g_{\sigma}}+(n-1) g_{\sigma}\right\|_{k-2, \alpha, \delta, r_{c}} \\
& =C\left\|\chi^{\prime \prime}+\frac{\chi^{\prime}}{r}+\frac{\chi}{r^{2}}\right\|_{k-2, \alpha, \delta} \\
& \leq C_{1} \frac{1}{|\sigma|^{n-1}}
\end{aligned}
$$

Note that one must be careful in the last line, since we are bounding Hölder norms, in which derivatives are calculated with respect to harmonic coordinates and not with respect to the coordinate $r$. But $r$ is related to the geodesic coordinate $s$ by

$$
r=O\left(e^{s}\right)
$$

for large $s$, and so we can establish the bound with respect to $s$ and then translate back into terms of $R$.

Thus, we have the following proposition:

Proposition 3.9. Let $(N, g)$ be a geometrically finite hyperbolic manifold, whose $l$ cusps all have toric cross-sections. Then, for any l-tuple $\sigma$ of geodesics, with the ith geodesic drawn from the ith cusp cross-section, it is possible to construct a manifold $\left(M_{\sigma}, g_{\sigma}\right)$ such that

$$
\left\|\Phi_{g_{\sigma}}\left(g_{\sigma}\right)\right\|_{k-2, \alpha, \delta, r_{c}}=O\left(|\sigma|^{1-n}\right) .
$$

These $\left(M_{\sigma}, g_{\sigma}\right)$ are $A H$ and have the same conformal infinity as $(N, g)$.

We will also need elliptic estimates for these operators. In analogy to the weighted Hölder norms, define the $\left(\delta, r_{c}\right)$-weighted $L^{\infty}$ norm to be $\|h\|_{L_{\delta, r_{c}}^{\infty}}=$ $\left\|e^{\delta r} \phi_{c}^{-1} h\right\|_{L^{\infty}}$.

Proposition 3.10. Let $L_{g}=D_{g} \Phi_{g_{\sigma}}$ be the linearization of $\Phi_{g_{\sigma}}$ at $g$, where $\left\|g-g_{\sigma}\right\|_{k, \alpha, \delta, r_{c}}<\epsilon_{0}$, and $\epsilon_{0}>0$ is chosen such that $L_{g}$ is elliptic. Then, there is some constant $\Lambda_{0}$, depending on $k, \alpha, \epsilon_{0}$ and $\delta$ such that we have the following estimate

$$
\|h\|_{k, \alpha, \delta, r_{c}} \leq \Lambda_{0}\left(\|L h\|_{k-2, \alpha, \delta, r_{c}}+\|h\|_{L_{\delta, r_{c}}^{\infty}}\right) .
$$


The proof of this is essentially that of the analogous estimate without the $r_{c}$ 's in [22]. The critical property of the weight functions $e^{-\delta r}$ and $\phi_{c}^{-1}$ that is required is that one can establish an estimate of the form

$$
\|f\|_{C^{2}} \leq C\|f\|_{C^{0}}
$$

with $C$ independent of the manifold and the choice of the $r_{c}$. Then, we can apply the standard Schauder estimate to the weighted functions, and the above estimate allows us to interchange the weight functions and the operator $L$. The point to note here is that $\Lambda_{0}$ does not depend in any way on $\sigma$ or $r_{c}$.

We end this section by quoting a key result of Graham and Lee's [21] on the behavior of the operator $L=D_{g_{\sigma}} \Phi_{g_{\sigma}}$. We know that $L$ is elliptic, but since we are working on noncompact manifolds $M$, it does not follow that $L$ is Fredholm. Thus, if we do not choose our function spaces carefully, $\operatorname{ker}(L)$ or coker $(L)$ could well be infinite-dimensional. Biquard's results give appropriate conditions to guarantee that $L$ is Fredholm (also see [23]).

Theorem 3.11 [21]. Let $\left(M^{n}, g_{0}\right)$ be an AH manifold. If $\delta \in(0, n-1)$, then

$$
L: S_{\delta}^{k, \alpha}\left(M^{n}\right) \longrightarrow S_{\delta}^{k-2, \alpha}\left(M^{n}\right)
$$

is Fredholm. Furthermore, $L$ is an isomorphism iff $\operatorname{ker}_{L^{2}}(L)=0$.

We will need to use both Lemma 3.6 and Theorem 3.11 below, so we will restrict $\delta$ to the interval $(n-1 / 2, n-1)$.

\section{Control of inverse}

In this section, we will perform the analysis which will allow us to invert our operators $\Phi_{g_{\sigma}}$. To do this, we will need to invert the linear operators $L_{\sigma}=2 D_{g_{\sigma}} \Phi_{g_{\sigma}}$ and get some kind of uniform control on the behavior of the inverses. We cannot get an absolute uniform bound on their operator norms, but we can make sure that they do not grow too fast with respect to the length of the Dehn surgery $\sigma$. We prove via a contradiction argument that we may choose $r_{c}$ such there exists a $\Lambda$ independent of $\sigma$ such that

$$
\|h\|_{k, \alpha, \delta, r_{c}} \leq \Lambda\left\|L_{\sigma}(h)\right\|_{k-2, \alpha, \delta, r_{c}}
$$

for all $h \in S_{\delta, r_{c}}^{k, \alpha}$, provided $k \geq 2$ and that $\sigma$ is large enough. 
The above estimate shows that $\operatorname{ker}\left(L_{\sigma}\right)=0$, and so by Theorem 3.11, we get that

$$
L_{\sigma}^{-1}: S_{\delta, r_{c}}^{k-2, \alpha} \longrightarrow S_{\delta, r_{c}}^{k, \alpha}
$$

is well defined and

$$
\left\|L_{\sigma}^{-1} f\right\|_{k, \alpha, \delta, r_{c}} \leq \Lambda\|f\|_{k-2, \alpha, \delta, r_{c}} .
$$

The proof of estimate (4.1) is essentially identical to Anderson's proof in the finite-volume case [1]. The only additional difficulty that we face here is that $S_{\delta, r_{c}}^{k, \alpha}$ functions are not necessarily in $L^{2}$, but as mentioned above, by choosing $\delta>n-1 / 2$, we can avoid this problem. In this section, any unlabeled norms will be assumed to be $L^{2}$ norms and $\delta \in(n-1 / 2, n-1)$ will be assumed to be fixed.

Proposition 4.1. Let $\left(M_{\sigma}, g_{\sigma}\right)$ be a sequence of approximate solutions. Then, there exist some $r_{c}$ and a constant $\Lambda$ independent of $\sigma$ such that

$$
\|h\|_{k, \alpha, \delta, r_{c}} \leq \Lambda\left\|L_{\sigma}(h)\right\|_{k-2, \alpha, \delta, r_{c}} .
$$

Proof. We work by contradiction, so we will have to take limits. This leads to some difficulty, since there is no uniform bound on the diameter of the $M_{\sigma}$ 's, and so the limits will not be uniquely defined. On the other hand, all of the limits are Einstein, which gives them extra structure which we will exploit.

Let us set up the contradiction. If there is no $\Lambda$ such that

$$
\|h\|_{k, \alpha, \delta, r_{c}} \leq \Lambda\left\|L_{\sigma}(h)\right\|_{k-2, \alpha, \delta, r_{c}}
$$

for all $\sigma$, then necessarily there is a sequence $h_{i} \in S_{\delta, r_{c}}^{k, \alpha}\left(M_{\sigma_{i}}, g_{\sigma_{i}}\right)$ such that

$$
\left\|h_{i}\right\|_{k, \alpha, \delta, r_{c}}=1
$$

but

$$
\left\|L_{i}\left(h_{i}\right)\right\|_{k-2, \alpha, \delta, r_{c}} \longrightarrow 0
$$

where we have replaced the subscript $\sigma_{i}$ by an $i$. 
By the Schauder [31] estimates, we know that there is a $\Lambda_{0}$ independent of $\sigma$ and $r_{c}$ such that

$$
\|h\|_{k, \alpha, \delta, r_{c}} \leq \Lambda_{0}\left(\left\|L_{\sigma}(h)\right\|_{k-2, \alpha, \delta, r_{c}}+\|h\|_{L_{\delta, r_{c}}^{\infty}}\right)
$$

for all $h \in S_{\delta, r_{c}}^{k, \alpha}\left(M_{\sigma}\right)$. Thus, under our assumption,

$$
\left\|h_{i}\right\|_{L_{\delta, r_{c}}^{\infty}} \geq \Lambda_{0}^{-1}>0 .
$$

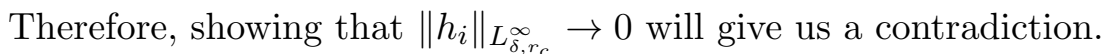

We will suppress the $r_{c}$ for the next while, since it will not play a role until later.

The most natural way of looking at $M_{\sigma}$ is to see it as being made up of two distinct pieces: the original hyperbolic manifold $N$ and the collection of black hole metrics with which we are filling in the cusps of $N$. Our strategy is to take the limit of the $h_{i}$ 's, which will lead to infinitesimal Einstein deformations of each piece. We will get our contradiction by showing that there can be no nontrivial deformations.

We will spend most of our time working on the filled regions of the cusps, i.e., the ends which are close to the black hole metrics. We will use the variables $r$ to refer to the $r$-variable in our parametrization of the black hole metrics, and $R$ will refer to the gluing region, as seen from the black hole metrics. Note that, by construction, $R=L(\sigma) / \beta_{1}$. Our analysis will take place on each black hole region separately, and so there will be no risk of confusion.

For the region associated to the $k$ th cusp, we have the relation

$$
R_{i}^{k}=\beta_{1} L\left(\sigma_{i}^{k}\right) \geq \beta_{1}\left|\sigma_{i}\right| .
$$

To begin, note that we have the following Weitzenböck formula [24]:

$$
\delta d h+d \delta h=D^{*} D h-R h+h \circ \text { ric },
$$

where $d$ is the exterior derivative on vector-valued one-forms induced by the connection, and $\delta$ is its formal adjoint.

From this, we get that

$$
\begin{aligned}
L h & =D^{*} D h-2 R h+h \circ \operatorname{ric}+\operatorname{ric} \circ h+2(n-1) h \\
& =\delta d h+d \delta h-R h+\operatorname{ric} \circ h+2(n-1) h .
\end{aligned}
$$

We will work primarily with this form of $L$, since we can prove stronger positivity properties with it. 
Now, by construction, we have that on $M_{i}$,

$$
\operatorname{ric}+(n-1) g=\tau(r)
$$

where $\tau$ is supported on the region of the black hole metric $\left(R_{i}-2, R_{i}-1\right)$ and satisfies

$$
|\tau| \leq C R_{i}^{1-n}
$$

Thus,

$$
L h=\delta d h+d \delta h-R h+(n-1) h+\tau \circ h .
$$

Now, consider

$$
\langle L h, h\rangle=\langle\delta d h, h\rangle+\langle d \delta h, h\rangle-\langle R h, h\rangle+(n-1)\|h\|^{2}+\langle\tau \circ h, h\rangle .
$$

Recall that we have $L h \rightarrow 0$ and $h$ bounded, and we want to show that $h \rightarrow 0$. This should be possible as long as all the terms on the right hand side are positive or tend toward 0 . Integration by parts will work on the first two terms, so we need to get a handle on the term $\langle R h, h\rangle$. We will do this by controlling the pointwise norm $(R h, h)$. First, we will break it up into three pieces. Let $h=h_{0}+(\operatorname{tr} h) g / n$ (so $h_{0}$ is the trace-free part of $h$ ).

\section{Lemma 4.2.}

$$
(R h, h)=\left(R h_{0}, h_{0}\right)+\mu_{i}(r)+O\left((\operatorname{tr} h)^{2}\right),
$$

where $\mu_{i}(r)$ is supported in the black hole region and is $O\left(R_{i}^{-(n-1)}\right)$.

Proof. To begin, note that

$$
\begin{aligned}
(R h, h) & =\left(R\left(h_{0}+\frac{(\operatorname{tr} h)}{n} g\right), h_{0}+\frac{(\operatorname{tr} h) g}{n}\right) \\
& =\left(R h_{0}, h_{0}\right)+\frac{\operatorname{tr} h}{n}\left(R g, h_{0}\right)+\frac{\operatorname{tr} h}{n}\left(R h_{0}, g\right)+\frac{(\operatorname{tr} h)^{2}}{n^{2}}(\text { ric }, g)
\end{aligned}
$$

Now, we have that (ric, $g)=s$, which is uniformly bounded. Furthermore, if we take an orthonormal frame $\left(e_{j}\right)$ which diagonalizes the curvature 
tensor,

$$
R h\left(e_{j}, e_{k}\right)=\sum_{l} h\left(R\left(e_{j}, e_{l}\right) e_{k}, e_{l}\right)
$$

Thus,

$$
\begin{aligned}
\left(R g, h_{0}\right) & =\left(g, R h_{0}\right) \\
& =\operatorname{tr}\left(R h_{0}\right) \\
& =\sum_{j, k} h_{0}\left(R\left(e_{j}, e_{k}\right) e_{j}, e_{k}\right) \\
& =\sum_{j, k} K_{j k} h_{0}\left(e_{k}, e_{k}\right) \\
& =-(n-1) \operatorname{tr}\left(h_{0}\right)+\mu_{i} \\
& =\mu_{i},
\end{aligned}
$$

where $\mu_{i}(r)=\sum_{j, k}\left(\delta_{j}^{k}+K_{j k}\right) h_{0}\left(e_{k}, e_{k}\right)=O\left(R_{i}^{-(n-1)}\right)$ since $h_{0}$ is uniformly bounded.

The following lemma allows us to control $\left(R h_{0}, h_{0}\right)$.

Lemma 4.3 [24]. Let

$$
a=\sup _{\left\{h_{0} \mid \operatorname{tr} h_{0}=0\right\}} \frac{\left(R h_{0}, h_{0}\right)}{\left|h_{0}\right|^{2}}
$$

be the largest eigenvalue of $R$ acting on $S_{0}^{2}$. Then,

$$
a<(n-2) K_{\max }-\text { ric }_{\min } .
$$

For the next term, we can use the following result from [1].

Lemma 4.4. As $i \rightarrow \infty$, we have $\left\|\operatorname{tr} h_{i}\right\|_{L^{2}} \longrightarrow 0$

Now, let $\mathcal{U}_{\rho}=\{x \mid r(x)<\rho\}$ be a tubular neighborhood of the totally geodesic core $T^{n-1}$ 's, and let $M_{i}^{\rho}=M_{i}-\mathcal{U}_{\rho}$. We will fix $\rho \leq R_{i}=\inf _{k} R_{i}^{k}$ below. Note that $\mathcal{U}_{\rho}$ has $q$ connected components, where $q$ is the number of cusps of $N$. 
By Lemmas 4.2 and 4.3 on $M_{i}^{\rho}$

$$
\begin{aligned}
(R(h), h) & =\left(R\left(h_{0}\right), h_{0}\right)+\mu_{i}+O\left((\operatorname{tr} h)^{2}\right) \\
& \leq\left((n-2) K_{\max }-\operatorname{ric}_{\min }\right)|h|^{2}+\mu_{i}+C_{1}|\operatorname{tr} h|^{2} \\
& =\left(-(n-2)+(n-1)+O\left(R_{i}^{1-n}\right)\right)|h|^{2}+\mu_{i}+C_{1}|\operatorname{tr} h|^{2} \\
& \leq\left(1+C_{2} R_{i}^{1-n}\right)|h|^{2}+\mu_{i}+C_{1}|\operatorname{tr} h|^{2} .
\end{aligned}
$$

Now consider

$$
\begin{aligned}
\int_{M^{\rho}}(L h, h) d V= & \int_{M^{\rho}}(\delta d h, h)+(d \delta h, h)-(R h, h) \\
& +(n-1)|h|^{2}+(\tau \circ h, h) d V \\
= & \int_{M^{\rho}}|\delta h|^{2}+|d h|^{2}-(R h, h)+(n-1)|h|^{2} \\
& +(\tau \circ h, h) d V+\int_{\partial \mathcal{U}_{\rho}} Q(h, \partial h) d A .
\end{aligned}
$$

Here, $Q(h, \partial h)$ is the boundary term from the integration by parts. It is a fixed quadratic polynomial in $h$ and its derivatives. Choose some $\epsilon>0$. By our estimates on $(R h, h)$ and assuming that $i$ is large enough that $\left|\tau_{i}\right|<\epsilon$ and

$$
1+C_{2} R_{i}^{1-n} \leq \frac{5 n}{12}
$$

we get that this last quantity is

$$
\begin{aligned}
\geq & \int_{M^{\rho}}-\left(\frac{5 n}{12}+\epsilon\right)|h|^{2}+(n-1)|h|^{2} d V-C_{1} \int_{M^{\rho}}(\operatorname{tr} h)^{2} d V \\
& -\int_{M^{\rho}} \mu_{i} d V+\int_{\partial \mathcal{U}_{\rho}} Q(h, \partial h) d A .
\end{aligned}
$$

Now, $\mu_{i}$ is $O\left(R_{i}^{1-n}\right)$ and supported on a region of bounded volume (the black hole region) and by the previous lemma $\left\|\operatorname{tr} h_{i}\right\|_{L^{2}} \longrightarrow 0$. So we may 
choose $i$ large enough that the previous quantity is

$$
\begin{aligned}
\geq & \int_{M^{\rho}}-\left(\frac{5 n}{12}+\epsilon\right)|h|^{2}+(n-1)|h|^{2} d V-2 \epsilon \\
& +\int_{\partial \mathcal{U}_{\rho}} Q(h, \partial h) d A \\
= & \left(\frac{7 n}{12}-1-\epsilon\right) \int_{M^{\rho}}|h|^{2} d V+\int_{\partial \mathcal{U}_{\rho}} Q(h, \partial h) d A-2 \epsilon .
\end{aligned}
$$

We also have that

$$
\int_{M^{\rho}}(L h, h) d V \leq \frac{1}{2}\left(\int_{M^{\rho}}|L h|^{2} d V+\int_{M^{\rho}}|h|^{2} d V\right)
$$

and

$$
\left|\int_{\partial \mathcal{U}_{\rho}} Q(h, \partial h) d A\right| \leq C V \operatorname{ol}\left(\partial \mathcal{U}_{\rho}\right)
$$

since we have $C^{k, \alpha}$ control over the $h_{i}$ 's.

Combining all this information gives

$$
\frac{1}{2} \int_{M^{\rho}}|L h|^{2} d V+C V \mathrm{ol}\left(\partial \mathcal{U}_{\rho}\right) \geq\left(\frac{7 n}{12}-\epsilon-\frac{3}{2}\right) \int_{M^{\rho}}|h|^{2} d V-2 \epsilon,
$$

where we can make $\epsilon>0$ arbitrarily small by making $i$ large.

By assumption, $\|L h\|_{L_{\delta}^{\infty}} \longrightarrow 0$. So we have that $\int_{M^{\rho}}|L h|^{2} d V \longrightarrow 0$. Remark that $\operatorname{inj}\left(\partial \mathcal{U}_{\rho}\right)=O\left(\frac{\rho}{R_{i}}\right)$. Thus, if we choose a sequence $\rho_{i}$ such that $\frac{\rho_{i}}{R_{i}} \longrightarrow 0$, we will obtain that $\int_{M^{\rho_{i}}}|h|^{2} d V$ tends to 0 . Since $S_{\delta}^{k, \alpha} \subset L^{2}$, this gives us uniform convergence of the $h_{i}$ 's on any set whose injectivity radius remains bounded below. Thus, by a diagonal argument, we can find some sequence $r_{i}$ such that $h_{i}$ converges uniformly to 0 on $M^{r_{i}}$ and $r_{i} / R_{i} \longrightarrow 0$. Note that we are finding a different $r_{i}$ for each cusp.

We will use these $r_{i}$ as the parameter for the cusp we are on in the weight function $\phi_{c}$. In other words, the $r_{i}$ obtained for the $j$ th cusp of the manifold $M_{i}$ will be the $r_{c}^{j}$ which determine the weight function on that cusp.

Now, let us examine what is happening on each component of $\mathcal{U}_{r_{i}}$, the complement of the set $M^{r_{i}}$. By construction, the core tori are collapsing to points, and so any neighborhood of these tori is degenerating to a line segment. Since we want to have a nice limit, we lift everything to finite 
covers in order to "unwrap the collapse." [7]. Choose a sequence of points $p_{i}$ in a core torus. Since $\operatorname{inj}(T(r))=O\left(r / R_{i}\right)$, by lifting $\left(\mathcal{U}_{r_{i}}, g_{i}, p_{i}\right)$ to an $\left[R_{i} / r_{i}\right]$-fold cover, where [ $]$ is the greatest integer part function, we will get a sequence of manifolds whose injectivity radius is bounded away from 0 .

By definition of the $S_{\delta, r_{c}}^{k, \alpha}$ norm, the $C^{k, \alpha}$-norm of these manifolds is bounded, so we get a limit manifold $\left(M_{\mathrm{BH}}, g_{\mathrm{BH}}\right)$. Clearly the $h_{i}$ 's lift to the finite covers too, and so we get lifted forms $\tilde{h}_{i}$. These $\tilde{h}_{i}$ satisfy

$$
\left\|\tilde{h}_{i}\right\|_{k, \alpha, \delta} \leq C
$$

on the lifted manifolds $\left(\tilde{\mathcal{U}}_{r_{i}}, \tilde{g}_{i}\right)$. Thus, given $\alpha^{\prime}<\alpha$, and $\delta^{\prime}<\delta$, we can extract a subsequence to get a limit $\tilde{h} \in S_{\delta^{\prime}}^{k, \alpha^{\prime}}$. Note that $\tilde{h}$ must be $T^{n-1}$ invariant and satisfy $L \tilde{h}=0$.

If $\left|r_{i}-r_{+}\right|$is uniformly bounded, the torus $T\left(r_{i}\right)$ stays within a fixed distance of the core torus for all $i$. Thus, the core torus can always see the region on which $h_{i}$ is tending uniformly to 0 . We can therefore take a pointed limit based at $p_{i} \in T\left(r_{i}\right)$ and conclude that $h=0$ if $r>r_{i}$. By analyticity of infinitesimal Einstein deformations, this leads us to conclude that $h$ is identically 0 , giving us our contradiction. Thus, we shall assume that $r_{i} \longrightarrow \infty$. This gives us that the limiting manifolds $\left(M_{\mathrm{BH}}, g_{\mathrm{BH}}\right)$ are complete. We are going to work at an infinite distance from the conformal infinity of $M_{\sigma}$, and so we can drop the weight factor $\delta$. It will also be understood that we are working on the lifted manifolds, and so we will suppress the tildes.

At this point, we would like to say that since $h_{i}\left(r_{i}\right) \longrightarrow 0$ and $r_{i} \longrightarrow \infty$, this forces our limiting sequence to have

$$
\lim _{r \rightarrow \infty}\|h\|=0 .
$$

We could then apply the following results to get our contradiction:

Proposition 4.5. $h$ is tangent to the space of $T^{n-1}$-invariant AHE metrics on $M$.

Proof. This is nontrivial, since spaces of AHE metrics are infinitedimensional, and so vector fields do not necessarily integrate. By [2], however, we know that if the AHE manifold $(M, g)$ has a $C^{2}$ conformal compactification, then infinitesimal deformations do indeed integrate. The function $\rho=r^{-1}$ is certainly 0 exactly at the conformal infinity of $\left(M_{\mathrm{BH}}, g_{\mathrm{BH}}\right)$, and 
with respect to the compactified metric,

$$
|d \rho|_{\bar{g}}^{2}=\left|\frac{d r}{r^{2}}\right|_{\bar{g}}^{2}=\frac{V(r)}{r^{2}}
$$

is nonzero on the boundary, and so $\rho$ is a defining function. Near the boundary, the compactified metric is

$$
\bar{g}=\frac{d r^{2}}{r^{2} V(r)}+\frac{V(r)}{r^{2}} d \theta^{2}+g_{T^{n-2}}
$$

One may replace $r$ by the coordinate $s$, where

$$
\frac{d s}{d r}=\frac{1}{r \sqrt{V(r)}} .
$$

To get

$$
\bar{g}=d s^{2}+F(s) d \theta^{2}+g_{T^{n-2}}
$$

a short calculation shows that $F$ is $C^{2}$ up to the boundary.

Proposition 4.6 ([25] c.f. also [26]). Let $g$ be a complete $T^{n-1}$-invariant AHE metric on the solid torus $D^{2} \times T^{n-2}$. Then $g$ is a black hole metric.

Proposition 4.7. Let $g_{t}$ be a curve of complete $T^{n-1}$-invariant AHE metric on the solid torus $D^{2} \times T^{n-2}$. Then $g_{t}$ is completely determined by $g_{0}$ and the curve $\gamma_{t}$ consisting of the conformal infinities of the $g_{t}$ 's.

Proof. By the previous proposition, all the $g_{t}$ 's are covered by $\left(D^{2} \times \mathbb{R}^{n-2}\right.$, $\left.\tilde{g}_{\mathrm{BH}}\right)$. Thus,

$$
g_{t}=\frac{\tilde{g}_{\mathrm{BH}}}{\Gamma_{t}},
$$

where $\Gamma_{t} \subset \operatorname{Isom}\left(g_{\mathrm{BH}}\right)$ is isomorphic to $\mathbb{Z}^{n-2}$. Consider the "defining function" $\rho=r^{-1}$ for $\tilde{g}_{\mathrm{BH}}$ (note that $\left(D^{2} \times \mathbb{R}^{n-2}, \rho^{2} \tilde{g}_{\mathrm{BH}}\right)$ is not a compact manifold with boundary).

Then the conformal infinity of $\left(D^{2} \times \mathbb{R}^{n-2}, \rho^{2} \tilde{g}_{\mathrm{BH}}\right)$ is a flat $S^{1} \times \mathbb{R}^{n-2}$. The action of $\Gamma_{t}$ commutes with multiplication by $\rho$, and so the conformal infinity of $g_{t}$ is the quotient of the flat $S^{1} \times \mathbb{R}^{n-2}$ by $\Gamma_{t}$. Conversely, the 
conformal infinity $\gamma_{t}$ of $g_{t}$ also determines the group $G_{t}=\Gamma_{t}+v_{t}$ up to conjugacy, where

$$
\gamma_{t}=\frac{\mathbb{R}^{n-1}}{G_{t}}
$$

Given an initial $g_{0}$, we can identify $v_{0}$ with the $S^{1}$ in the universal cover. This determines $v_{t}$ for $t>0$, and thus $\Gamma_{t}$ determines $g_{t}$.

Combining these propositions gives us the following corollary:

Corollary 4.8. A $T^{n-1}$-invariant $A H E$ metric on a solid torus has no nontrivial infinitesimal deformations for which

$$
\lim _{r \rightarrow \infty}|h(r)|=0 \text {. }
$$

Unfortunately (for us), we cannot apply these propositions directly; the problem comes from the fact that the $h_{i}$ 's tend to $h$ uniformly on compact subsets, and we cannot relate the rate of convergence to the size of the compact set. Thus, we cannot know that $r_{i}$ is included in each set. Consider the following example: Let $I_{k}=[-k, k]$, and

$$
\begin{array}{r}
f_{k}: I_{k} \longrightarrow[-1,1] \\
x \mapsto \frac{x}{k} .
\end{array}
$$

Clearly, $\left\|f_{k}^{\prime}(x)\right\|_{L^{\infty}} \longrightarrow 0$ as $k \longrightarrow \infty$, and so one would expect that the $f_{k}$ 's are tending toward a constant function. This constant may, however, depend on the basepoint $x_{k}$; let $x_{k}=\alpha k$, where $-1<\alpha<1$. Then the (pointed) Gromov-Hausdorff limit of the triple $\left(I_{k}, x_{k}, f_{k}(x)\right)$ will be the triple $(\mathbb{R}, 0, \alpha)$. Thus the limit of the $f_{k}$ is indeed a constant function, but the constant depends on the choice of the basepoints $x_{k}$.

The issue here is that the manifolds $I_{k}$ are converging to their limit faster than the $f_{k}$ are converging to theirs, and so the convergence cannot be made uniform on the whole set.

More precisely, we have that

$$
\begin{aligned}
\left|f_{k}(x)-f_{k}(y)\right| & \leq\left|\int_{x}^{y} f_{k}^{\prime}(s) d s\right| \\
& \leq\left\|f_{k}^{\prime}\right\|_{L^{\infty}}\left|\int_{x}^{y} d s\right| \\
& \leq\left\|f_{k}^{\prime}\right\|_{L^{\infty}}|x-y| .
\end{aligned}
$$


Therefore, if we choose points $x_{k}, y_{k}$ whose distance is increasing fast enough, we cannot conclude that $f_{k}\left(x_{k}\right)$ and $f_{k}\left(y_{k}\right)$ have the same limit.

On the other hand, if we require that $\left\|f_{k}^{\prime}\right\|_{L^{\infty}} \longrightarrow 0$ more rapidly than any two points can separate, say by demanding that $\operatorname{diam}\left(I_{k}\right)\left\|f_{k}^{\prime}\right\|_{L^{\infty}} \longrightarrow 0$, then we can get that

$$
\begin{aligned}
\left|f_{k}(x)-f_{k}(y)\right| & \leq\left\|f_{k}^{\prime}\right\|_{L^{\infty}}|x-y| \\
& \leq \operatorname{diam}\left(I_{k}\right)\left\|f_{k}^{\prime}\right\|_{L^{\infty}} \longrightarrow 0
\end{aligned}
$$

no matter which basepoints we take.

Let us now attempt to adapt this argument to the operator $L$. We will start by analyzing the limiting case to motivate our choice of the weight $\phi_{c}$, and then we will get our hands dirty with the actual estimates that we need to finish our proof. As we will see below, on $T^{n-1}$-invariant deformations $h$, the components of $L h=0$ are asymptotic to Euler equations. For the components $(L h)_{1 j}$, this equation has a nontrivial 0 th order term, and so all of its solutions either blow up or decay to 0. Its other components are asymptotic to equations of the form

$$
L f=r^{2} f^{\prime \prime}+n r f^{\prime}=0
$$

which have constant solutions. This leads to a problem for us, since we could have the same situation as above; even though $f\left(r_{i}\right) \rightarrow 0$ and $f$ tends to a constant, we cannot conclude that $f \longrightarrow 0$ everywhere. This is where the weight $\phi_{c}$ in our norms comes in.

By use of an integrating factor [27], we may rewrite this as

$$
f(r)=\int \frac{1}{r^{n}} \int s^{n-2} L f(s) d s d r
$$

Thus,

$$
\begin{aligned}
\left|f\left(r_{1}\right)-f\left(r_{2}\right)\right| & =\left|\int_{r_{1}}^{r_{2}} \frac{1}{r^{n}} \int_{r_{+}}^{r} s^{n-2} L f(s) d s d r\right| \\
& \leq \frac{\|L f\|_{L^{\infty}}}{n-1}\left|\int_{r_{1}}^{r_{2}} r^{-1}+O\left(r^{1-n}\right) d r\right| \\
& \leq \frac{\|L f\|_{L^{\infty}}}{n-1}\left|\log \left(\frac{r_{2}}{r_{1}}\right)+C_{0}\right| \\
& \leq C_{1} \frac{R_{i}}{r_{i}}\|L f\|_{L^{\infty}} \\
& \leq C_{1}\left\|\phi_{c}^{-1} L f\right\|_{L^{\infty}} \longrightarrow 0 .
\end{aligned}
$$


As we remarked above, our situation is a bit more delicate, since we are not working exactly with this operator, but with perturbations of it. Furthermore, since we need to use the rate at which the $h_{i}$ 's converge, we cannot just work with limits, but must rather get precise bounds on how things behave asymptotically.

We now want to analyze the system of ODEs that these deformations must satisfy. The proof of the following proposition consists of a long calculation which is quite complicated, but fairly straightforward. The interested reader may consult [26] to see the gory details, and the masochistic reader may attempt it for her- or himself.

Proposition 4.9. Say we have a black hole metric g. Let $e_{1}=\sqrt{V} \partial_{r}$, $e_{2}=\frac{1}{\sqrt{V}} \partial_{\phi_{j}}$ and $e_{j}=\frac{1}{r} \partial_{\phi_{j}}$, where the $\partial_{\phi_{j}}$ 's, $3 \leq j \leq n$ form an orthonormal basis for the core torus. Then, if $h$ is $S^{1} \times T^{n-2}$-invariant, $L h=2 D_{g} \Phi_{g} h$ is given by

$$
\begin{aligned}
(L h)_{11}= & A h_{11}+h_{11}\left(\frac{\left(V^{\prime}\right)^{2}}{2 V}+\frac{2(n-2) V}{r^{2}}\right)-h_{22}\left(\frac{\left(V^{\prime}\right)^{2}}{2 V}+2 K_{12}\right) \\
& -2 \sum_{k>2}\left(\left(\frac{V}{r^{2}}\right)+K_{1 k}\right) h_{k k} \\
(L h)_{22}= & A h_{22}+h_{22} \frac{\left(V^{\prime}\right)^{2}}{2 V}-h_{11}\left(\frac{\left(V^{\prime}\right)^{2}}{2 V}+2 K_{12}\right)-2 \sum_{k>2} K_{2 k} h_{k k} \\
(L h)_{12}= & A h_{12}+h_{12}\left(\frac{\left(V^{\prime}\right)^{2}}{V}+\frac{2(n-2) V}{r^{2}}+2 K_{12}\right)
\end{aligned}
$$

where

$$
A h_{i j}=\left(-V h_{i j}^{\prime \prime}-\left(V^{\prime}+\frac{n-2}{r} V\right) h_{i j}^{\prime}\right)
$$

If $j>2$, we have

$$
\begin{aligned}
& (L h)_{j j}=A h_{j j}+\frac{2 V}{r^{2}}\left(h_{j j}-h_{11}\right)-2 \sum_{k \neq j} K_{k j} h_{k k} \\
& (L h)_{1 j}=A h_{1 j}+h_{1 j}\left(\frac{\left(V^{\prime}\right)^{2}}{4 V}+\frac{(n+1) V}{r^{2}}+2 K_{1 j}\right) \\
& (L h)_{2 j}=A h_{2 j}+h_{2 j}\left(\frac{\left(V^{\prime}\right)^{2}}{4 V}+\frac{V}{r^{2}}+2 K_{2 j}\right),
\end{aligned}
$$


and finally, if $i, j>2$, we get

$$
(L h)_{i j}=A h_{i j}+h_{i j}\left(\frac{\left(V^{\prime}\right)^{2}}{2 V}+2 K_{i j}\right) .
$$

This system seems unmanageable for the black hole metrics, but we can get around this by noting the following two facts:

Proposition 4.10. Let $g_{C}$ be a complete hyperbolic cusp metric

$$
g_{C}=r^{-2} d r^{2}+g_{T^{n-1}}
$$

where $g_{T^{n-1}}$ is an arbitrary flat metric on the torus with orthonormal basis $\partial_{\phi_{j}}, 2 \leq j \leq n$. Let $\left(e_{1}=r \partial_{r}, e_{j}=\partial_{\phi_{j}} / r ; j \geq 2\right)$ form an orthonormal frame for $g_{C}$. Then, if $h$ is $T^{n-1}$-invariant, $L_{C} h=L_{g_{C}} h$ is given by the following formulae.

$$
(L h)_{11}=A h_{11}+2(n-1) h_{11}
$$

and if $j, k>2$,

$$
\begin{aligned}
& (L h)_{j j}=A h_{j j}+2 \operatorname{tr} h-2 h_{11} \\
& (L h)_{1 j}=A h_{1 j}+n h_{1 j} \\
& (L h)_{j k}=A h_{j k},
\end{aligned}
$$

where $A=-r^{2} \partial_{r}^{2}-n r \partial_{r}$.

Proof. Set $V(r)=r^{2}$ and $K_{j k}=-1+\delta_{j}^{k}$ above.

Now that we have the much simpler form of $L$ for $g_{C}$, we must relate it to the corresponding operator on the black hole metrics. We will use $C^{k}$ estimates instead of Hölder ones, since they are easy to establish, and we do not need the stronger norms; we already have the existence of the limit form $h$. The $C^{k}$ norms will be calculated in the same harmonic coordinates as the $S_{\delta}^{k, \alpha}$ ones. An easy calculation gives:

Proposition 4.11. If $\|h\|_{C^{2}}$ is bounded and $T^{n-1}$-invariant, then

$$
\left\|L_{C} h-L_{\mathrm{BH}} h\right\|_{L^{\infty}}=O\left(r^{-(n-1)}\right) .
$$


Thus, if $h$ is $T^{n-1}$-invariant and bounded in $C^{2}$, we get the following systems of equations for $L h=0$ :

$$
\begin{aligned}
A h_{11}+2(n-1) h_{11} & =u_{11} \\
A h_{j j}+2 \operatorname{tr} h-2 h_{11} & =u_{j j} \\
A h_{1 j}+n h_{1 j} & =u_{1 j} \\
A h_{i j} & =u_{i j}
\end{aligned}
$$

where $i, j>1$, all the components of $h$ are bounded and $|u|=O\left(r^{-(n-1)}\right)$.

Recall that we want to prove that

$$
\lim _{r \rightarrow \infty}|h(r)|=0
$$

This is straightforward in the case of the components $h_{11}, h_{1 j}$ and $h_{j j}$. All of these satisfy nonhomogeneous Euler equations with nonzero indicial roots. One can write out the solutions to the nonhomogeneous equations in terms of the fundamental solutions using variations of parameters [27] and see that bounded solutions must tend to 0 as $r \rightarrow \infty$.

Thus, all that is left is to examine the equations for $h_{i j}, i, j>1$. Although the equation that they satisfy seems to be the simplest of the ones that we have looked at, the $h_{i j}$ 's are in fact the most subtle case. As we mentioned above, the issue is that the equation $A h_{i j}=0$ is an Euler equation with no 0th order term. Therefore, there are constant solutions, which neither blow up nor go to zero as $r \rightarrow \infty$.

Since we want to invoke the rate at which $L h_{i}$ tends toward 0 , we will now be working with the $h_{i}$ 's rather than $h$. Thus, we will need to quantify the rate at which the $h_{i}$ 's are converging to their $T^{n-1}$-invariant limit.

Let

$$
\hat{h}_{i}(r)=\frac{1}{A(T(r))} \int_{T(r)} h_{i}(r, x) d A
$$

be the average of $h$ over the torus $T(r)$. Then, we have

Proposition 4.12. $\left\|h_{i}-\hat{h}_{i}(r)\right\|_{C^{2}}=O\left(\frac{r}{R_{i}}\right)$.

Proof. We have

$$
\sup _{x \in T(r)}\left|h_{i}(r, x)-\hat{h}_{i}(r)\right| \leq \frac{1}{A(T(r))} \int_{T^{n-1}(r)}\left|h_{i}(r, x)-\hat{h}_{i}(r)\right| d A .
$$


Now, we know that $h_{i}$ is the lift of a form defined on a torus of diameter $O\left(\frac{r}{R_{i}}\right)$. Since $\left\|h_{i}\right\|_{k, \alpha} \leq C$, we know that the integrand must be less than $C$ times the diameter of the base torus, and so it is also $O\left(\frac{r}{R_{i}}\right)$. We have assumed that $k \geq 3$, and so we can repeat this for the first and second derivatives of $h_{i}$.

We know that on our unwrapped black hole metrics, we have

$$
L(\hat{h})=L_{C}(\hat{h})+O\left(r^{-(n-1)}\right) .
$$

Finally, since $\|L h\|_{L_{r_{c}}^{\infty}}=\left\|\phi_{c}^{-1} L h\right\|_{L^{\infty}} \rightarrow 0$,

$$
L(h)=o\left(\phi_{c}\right) \text {. }
$$

Putting this all together gives us

$$
\begin{aligned}
L_{C}\left(\hat{h}_{i}\right) & =L_{\mathrm{BH}}\left(\hat{h}_{i}\right)+O\left(r^{-(n-1)}\right) \\
& =L_{\mathrm{BH}}\left(h_{i}\right)+O\left(r^{-(n-1)}\right)+O\left(\frac{r}{R_{i}}\right) \\
& =o\left(\phi_{c}\right)+O\left(r^{-(n-1)}\right)+O\left(\frac{r}{R_{i}}\right) .
\end{aligned}
$$

Now, dropping the $i$ 's and the hats, we see that if $a, b>1$

$$
r^{2} h_{a b}^{\prime \prime}+n r h_{a b}^{\prime}=e_{a b},
$$

where

$$
e_{a b}=O\left(\frac{r}{R_{i}}\right)+O\left(r^{-(n-1)}\right)+o\left(\phi_{c}\right) \text {. }
$$

Recall that we know that $\lim _{i \rightarrow \infty} h_{i}\left(r_{i}\right)=0$. We want to show that this is true for any sequence $\rho_{i} \leq r_{i}$ with $\rho_{i} \rightarrow \infty$.

Using an integrating factor, we get

$$
\begin{aligned}
\left|h_{a b}\left(r_{i}\right)-h_{a b}\left(\rho_{i}\right)\right| \leq & \int_{\rho_{i}}^{r_{i}} \frac{1}{r^{n}} \int_{r+}^{r}\left|e_{a b}(s)\right| s^{n-2} d s d r \\
\leq & \int_{\rho_{i}}^{r_{i}} \frac{1}{r^{n}}\left(C_{1} \int_{r+}^{r} \frac{s^{n-1}}{R_{i}} d s+C_{2} \int_{r+}^{r} s^{-1} d s\right. \\
& \left.+c_{i} \int_{r+}^{r} s^{n-2} \phi_{c}(s) d s\right) d r
\end{aligned}
$$


where $c_{i} \rightarrow 0$. Then, this is

$$
\leq C_{3} \frac{r_{i}}{R_{i}}+C_{4} r_{i}^{-(n-1)}+c_{i} \frac{r_{i}}{R_{i}}
$$

Thus, we can conclude that

$$
\lim _{r \rightarrow \infty}|h(r)|=0
$$

So by Corollary 4.8, we finally have our contradiction and therefore the main estimate.

The reader may be somewhat uneasy at the following aspect of the above proof: the fact that the distance between a sequence of pairs of points could grow to infinity within a filling region caused us some difficulty at getting a uniform control on $h$ on the entire filling region. Should this problem not become even more serious when comparing points in different filling regions? The answer is no, since the weight functions are defined locally on each filling region and are fine-tuned to the size of each one.

Finally, to finish this chapter, we extend the invertibility of $L_{g}=2 D_{g} \Phi_{g_{\sigma}}$ to a neighborhood of our approximate solution. Below, $B(x, \epsilon)$ will refer to a ball in the $S_{\delta, r_{c}}^{k, \alpha}$-topology, for the same choices of $k, \alpha, \delta$ and $r_{c}$ as above.

Proposition 4.13. There exist $\epsilon>0, \Lambda>0$ such that for all $\sigma$ large enough, we can choose $r_{c}$ such that the operator $L_{g}$ is invertible on the ball $B\left(g_{\sigma}, \epsilon\right)$, and for all $f \in \Phi\left(B\left(g_{\sigma}, \epsilon\right)\right)$, we have that

$$
\left\|\left(L_{g}\right)^{-1} f\right\|_{k, \alpha, \delta, r_{c}} \leq \Lambda\|f\|_{k-2, \alpha, \delta, r_{c}} .
$$

Proof. If not, there is a sequence of $g_{i}$ 's and $\sigma_{i}$ 's with $\left\|g_{i}-g_{\sigma_{i}}\right\|_{k, \alpha, \delta, r_{c}} \rightarrow 0$, and a sequence $h_{i} \in S_{\delta, r_{c}}^{k, \alpha}\left(M_{i}, g_{i}\right)$ such that

$$
\left\|h_{i}\right\|_{k, \alpha, \delta, r_{c}}=1
$$

but

$$
\left\|L_{g_{i}} h_{i}\right\|_{k-2, \alpha, \delta, r_{c}} \longrightarrow 0
$$

But then we can repeat the proof of the previous proposition to obtain a contradiction. 


\section{Conclusions}

In this section, we conclude the construction of the AHE metrics on the $M_{\sigma}$ 's.

Proposition 5.1. If $|\sigma|$ is large enough, then the manifold $M_{\sigma}$ admits an AHE manifold with the same conformal infinity as $N$.

Proof. Let $\delta \in\left(\frac{n-1}{2}, n-1\right)$. There is some $\epsilon>0$ such that for all $g$ in the ball $B\left(g_{\sigma}, \epsilon\right)$, the map

$$
\Phi_{g_{\sigma}}: S_{\delta, r_{c}}^{k, \alpha}(g) \longrightarrow S_{\delta, r_{c}}^{k-2, \alpha}(g)
$$

has an invertible linearization and

$$
\left\|(D \Phi)^{-1} f\right\|_{k, \alpha, \delta, r_{c}} \leq \Lambda\|f\|_{k-2, \alpha, \delta, r_{c}}
$$

Thus, by the inverse function theorem [28], $\Phi$ is invertible on $B\left(g_{\sigma}, \epsilon\right)$ and maps $B\left(g_{\sigma}, \epsilon\right)$ surjectively onto some $\mathcal{U} \subset S_{\delta, r_{c}}^{k-2, \alpha}$ containing $\Phi_{g_{\sigma}}\left(g_{\sigma}\right)$. Thus, we need to show that $0 \in \mathcal{U}$. To do this, we will need a lower bound on the diameter of $\mathcal{U}$.

Let $B\left(\Phi\left(g_{\sigma}\right), \gamma\right) \subset \mathcal{U}$. By our control on $(D \Phi)^{-1}$, we know that $\Phi^{-1}$ is Lipschitz with Lipschitz constant $\Lambda$. Therefore,

$$
\Phi^{-1}\left(B\left(\Phi\left(g_{\sigma}\right), \gamma\right)\right) \subseteq B\left(g_{\sigma}, \Lambda \gamma\right)
$$

Thus, if we choose $\gamma=\frac{\epsilon}{\Lambda}$, we will obtain that $B\left(\Phi\left(g_{\sigma}\right), \gamma\right) \subseteq \operatorname{Im}(\Phi)$. All that is left to do to show the existence of a solution to $\Phi=0$ is to make sure that $0 \in B\left(\Phi\left(g_{\sigma}\right), \gamma\right)$ for $\sigma$ large enough. But $\left\|\Phi\left(g_{\sigma}\right)\right\|_{k-2, \alpha, \delta, r_{c}}=O\left(|\sigma|^{1-n}\right)$, and so

$$
\left\|\Phi\left(g_{\sigma}\right)-0\right\|_{k-2, \alpha, \delta, r_{c}} \leq C|\sigma|^{1-n} \leq \frac{\epsilon}{\Lambda}
$$

for $|\sigma|$ large enough, since $\epsilon$ and $\Lambda$ are fixed.

Let $g_{\mathrm{E}}=\Phi^{-1}(0)$. Since $g_{\sigma}$ has negative Ricci curvature, we may assume that $\epsilon$ is small enough that $g_{\mathrm{E}}$ does too. Since $B_{g_{\sigma}}\left(g_{\sigma}\right)=0$, and $\lim _{r \rightarrow \infty} \| g-$ $g_{\sigma} \|_{k, \alpha, \delta, r_{c}}=0$ we can conclude that $\lim _{r \rightarrow \infty}\left|B_{g_{\sigma}}\left(g_{\mathrm{E}}\right)\right|=0$. Invoking Lemma 3.8 gives us that $g_{\mathrm{E}}$ is an Einstein metric. Since $g_{\mathrm{E}}$ is a perturbation of $g_{\sigma}$ by an element of $S_{\delta, r_{c}}^{k, \alpha}$, it must have the same conformal infinity, so we are done. 
Note that by construction, all of these metrics are nondegenerate, i.e., $D \Phi$ has no $L^{2}$ kernel. Furthermore, all of these metrics are isolated points in the moduli space of AH Einstein metrics on $M_{\sigma}$ with boundary metric $[\gamma]$, equipped with the $C_{\delta}^{k, \alpha}$ topology.

Abusing notation slightly, let us denote these AHE metrics by $g_{\sigma}$. Then we have that, for any sequence of points $p_{\sigma}$ which remain within a bounded distance of a gluing torus

$$
\lim _{|\sigma| \rightarrow \infty}\left(M_{\sigma}, g_{\sigma}, p_{\sigma}\right)=(N, g)
$$

in any of the $S_{\delta}^{k, \alpha}$ topologies, where $(N, g)$ is our original hyperbolic manifold. By only opening one cusp at a time, we will construct nonhyperbolic AHE manifolds with cusps.

Proof of Theorem 1.7. Let $\sigma=\left(\sigma_{1}, \ldots, \sigma_{l}\right)$ have large enough norm that the manifold $M_{\sigma}$ admits an Einstein metric. Then, define a sequence $\sigma_{i}=$ $\left(v_{i}, \sigma_{2}, \ldots, \sigma_{l}\right)$, where $v_{i}$ is a sequence of geodesics such that $L\left(v_{i}\right) \rightarrow \infty$. Let $p_{i}$ be a sequence of points within a bounded distance of a given gluing torus. Then,

$$
\left(M, g_{\infty}\right)=\lim _{i \rightarrow \infty}\left(M_{i}, g_{i}, p_{i}\right)
$$

is a nonhyperbolic AHE manifolds with cusps. The conformal infinity of $\left(M, g_{\infty}\right)$ is the same as that of $(N, g)$.

All of the conformal infinities involved in our constructions are necessarily conformally flat, since they are the conformal infinity of a hyperbolic manifold. All of the examples known to the author of conformal classes bounding infinitely many AHE manifolds are conformally flat.

It is possible to extend this filling construction to some other types of finite-volume cusp ends. In such a case, the cusp cross-section is necessarily a compact flat manifold and thus a finite quotient of a torus by Bieberbach's theorem. One can find sufficient conditions to construct an approximate solution in terms of the geometry of the flat cross-section. In all dimensions greater than 3 , there exist flat cusp cross-sections to which this procedure cannot be applied. For more on this, see [1].

\section{Acknowledgments}

This work was part of the author's doctoral thesis at Stony Brook University, written under the supervision of Professor Michael Anderson. I would like 
to thank him for his guidance, advice and support. The article was written during a postdoctoral fellowship at the CRM in Montreal supported in part by Professors Vestislav Apostolov of UQAM and Niky Kamran of McGill, and I would like to thank them for their support, both moral and financial. I would additionally like to express my gratitude to all my friends and family, and especially to Jean Dendy and Louis Garceau, for their encouragement. I am also very grateful to the referees for their comments and suggestions. Finally, I would like to dedicate this article to the memory of Ray "Rocco" Golbert, whose support and encouragement helped make my time in graduate school much easier, and who will be sorely missed.

\section{References}

[1] M. Anderson, Dehn filling and Einstein metrics in higher dimensions, Preprint, Arxiv.org/math.DG/0303260.

[2] M. Anderson, Einstein metrics with prescribed conformal infinity on 4-manifolds, Preprint, Arxiv.org/math.DG/0105243.

[3] E. Witten, Anti de Sitter space and holography, Adv. Theor. Math. Phys. 2 (1998), no. 2, 253-291.

[4] M. Anderson, Geometric aspects of the AdS/CFT correspondence, AdS/CFT Correspondence: Einstein Metrics and Their Conformal Boundaries, Euro. Math. Soc., Zurich, 2005, 1-31.

[5] J. Bekenstein, Information in the holographic universe. Sci. Amer. 289 (2003), no. 2, 58-65.

[6] M. Anderson, Topics in conformally compact Einstein metrics, Proceedings of the CRM short programme on Riemannian Geometry, Preprint, Arxiv.org/math.DG/0503243.

[7] M. Anderson, Boundary regularity, uniqueness and non-uniqueness for AH Einstein metrics on 4-manifolds. Adv. Math. 179 (2003), no. 2, 205-249.

[8] M. Kapovich, Hyperbolic manifolds and discrete groups, Progress in Mathematics, 183, Birkhäuser Boston, Inc., Boston, MA, 2001.

[9] F. Bonahon and J.-P. Otal, Vari/ét/és hyperboliques à géodésiques arbitrairement courtes, Bull. Lond. Math. Soc. 20 (1988), no. 3, 255-261. 
[10] C. Taubes, Self-dual Yang-Mills connections on non-self-dual 4-manifolds, J. Diff. Geom. 17 (1982), no. 1, 139-170.

[11] N. Kapouleas, Complete constant mean curvature surfaces in Euclidean three-space. Ann. of Math. (2) 131 (1990), no. 2, 239-330.

[12] R. Mazzeo, D. Pollack, and K. Uhlenbeck, Connected sum constructions for constant scalar curvature metrics, Topol. Method. Nonlinear Anal. 6 (1995), no. 2, 207-233.

[13] R. Mazzeo and F. Pacard, Maskit combinations of Poincare-Einstein metrics, Preprint, Arxiv.org/math.DG/0211099.

[14] W. Thurston, Three-dimensional geometry and topology, Vol. 1, in Princeton Mathematical Series, ed. Silvio Levy, 35, Princeton University Press, Princeton, NJ, 1997.

[15] S. Bleiler and C. Hodgson, Spherical space forms and Dehn filling. Topology 35 (1996), no. 3, 809-833.

[16] M. Gromov and W. Thurston, Pinching constants for hyperbolic manifolds, Invent. Math. 89 (1987), no. 1, 1-12.

[17] P. Petersen, Riemannian geometry, Graduate Texts in Mathematics, 171, Springer-Verlag, New York, 1998.

[18] J. Ratcliffe, On the isometry groups of hyperbolic manifolds, The Mathematical Legacy of Wilhelm Magnus: Groups, Geometry and Special Functions (Brooklyn, NY, 1992), 491-495, Contemp. Math., 169, Amer. Math. Soc., Providence, RI, 1994.

[19] M. Anderson, Convergence and rigidity of manifolds under Ricci curvature bounds, Invent. Math. 102 (1990), no. 2, 429-445.

[20] D. Gilbarg and N. Trudinger, Elliptic partial differential equations of second order, Second Edition, Grundlehren der Mathematischen Wissenschaften, 224, Springer-Verlag, Berlin, 1983.

[21] R. Graham and J. Lee, Einstein metrics with prescribed conformal infinity on the ball, Adv. Math. 87 (1991), no. 2, 186-225.

[22] J. Lee, Fredholm operators and Einstein metrics on conformally compact manifolds, Preprint, Arxiv.org/math.DG/0105046.

[23] O. Biquard, Métriques d'Einstein asymptotiquement symétriques, Astérisque (2000), no. 265. 
[24] A. Besse, Einstein manifolds, Ergebnisse der Mathematik und ihrer Grenzgebiete (3), 10, Springer-Verlag, Berlin, 1987.

[25] L. Berard-Bergery, Sur de nouvelles variétés riemanniennes d'Einstein, 6, Inst. Élie Cartan, Univ. Nancy, Nancy, 1982, 1-60.

[26] G. Craig, Dehn filling and asymptotically hyperbolic manifolds, Stony Brook University, Thesis, 2005, http://cs.ubishops.ca/ gcraig/phd.ps, http://cs.ubishops.ca/ gcraig/phd.pdf

[27] W. Boyce and R. DiPrima, Elementary Differential Equations and Boundary Value Problems, John Wiley \& Sons, Inc., New YorkLondon-Sydney, 1965.

[28] J. Dieudonné, Foundations of modern analysis. Pure and Applied Mathematics, X, Academic Press, New York-London, 1960.

[29] M. Anderson, Some results on the structure of conformally compact Einstein metrics, Preprint, Arxiv.org/math.DG/0402198.

[30] J. M. Feld, A kinematic characterization of lineal elements in the plane and of their differential invariants under the group of whirl-similitudes and some of its subgroups, Amer. J. Math. 70 (1948), 129-138.

[31] J. Jost, Partial differential equations. Graduate Texts in Mathematics, 214, Springer-Verlag, New York, 2002.

Bishop's UNIVERSITY

LENNOXVILLE

QUEBEC

CANADA

E-mail address: gcraig@ubishops.ca

Received August 19, 2005 